\title{
XXXVIII. Viburni generis species varietatesque asiaticae nuper pro novis anglice descriptae.
}

\author{
Auctore Alf jeal Pehder.
}

\section{6, 1908.}

Sect. Thyrsosma Rehder in Sargent, Trees and Shrubs, II,

$\mathrm{Ab}$ omnibus aliis sectionibus inflorescentiis paniculatis bene distincta; putamen ventre profunde sulcatum. (Thyrsosma Rafinesque, Sylv. Tellur., 130, 1838.)

1. Viburnum yunnanense Rehder, I. c., p. 107.

Frutex (vel arbor?) ramulis hornotinis dense fasciculato-pubescentibus, annotinis flavo-cinereis, vetustioribus cinereo-brunneis. Gemmae 4-perulatae. Folia decidua ovalia vel ovali-oblonga v. oblongo-obovata, obtusa, basi rotundata vel attenuata, crenato-serrata, supra obseure viridia, glabra venis sparse pilosis exceptis, subtus pallidiora, glandulis minutis albidis conspersa, ad venas fasciculato-pilosa, utrinque 5-6costata costis rectis in dentes exeuntibus, $3-6 \mathrm{~cm}$ longa (nondum perfecte matura); petioli $1 \mathrm{~cm}$ longi, dense fasciculato-pubescentes. Paniculae terminales et laterales in apice ramulorum bifoliorum pedunculo $2-3 \mathrm{~cm}$ longo gestae, fere hemisphericae et satis densae. $3-5 \mathrm{~cm}$ diam.. fasciculato-pubescentes ramulis bracteis lanceolatis ciliatis suffultis; ovarium sparse glandulosum, calycis dentes orbiculato-ovati, sparse ciliati; corolla rotata, glabra, $5 \mathrm{~mm}$ diam., lobis orbiculato-ovatis; stamina corollam non superantia; stylus crassus brevisque, calycis dentes aequans. Fructus desiderantur.

Yunnan: Mengtze, $2300 \mathrm{~m}$ alt., A. Henry no. 11015 (in herb. Arnold Arb.).

Proximum Viburno Sieboldii quod praecipue foliis majoribus, 8-12costatis, paniculis majoribus sparsius pubescentibus v. glabrescentibus, ramulis floriferis 4 -6-foliatis differt.

Sect. Lantana De Candolle.

2. Viburnum hypoleucum Rehder, l, c., p. 111.

Frutex $1 \mathrm{~m}$ altus ramulis hornotinis laxe stellato-tomentosis, mox glabrescentibus, annotinis pallide flavo-brunneis, lenticellis parvis sparsis instructis. Gemmae nudae. Folia decidua, lanceolata-oblonga v, anguste oblonga, acuta v. obtusiuscula, basi rotundata plerumque obliqua v. interdum subcordata, integra v. remote denticulata, supra obscure viridia, glabra, subtus dense albo-tomentosa, 7-10-costata costis curvatis et anastomosantibus, supra leviter impressis, subtus elevatis, 5-10 cm longa et $1,5-4 \mathrm{~cm}$ lata; petioli $1 \mathrm{~cm}$ longi, stellato-tomentosi. Corymbi terminales dense stellato-tomentosi, $4-5,5 \mathrm{~cm}$ diam., pedunculo $2.5 \mathrm{~cm}$ longo; radii plerumque 7 , fere aequilongi, flores breviter pedicellati, in radiis secundi $\mathbf{v}$. tertii ordinis bracteis lineari-lanceolatis suffulti, ovarium oblongo-ovoideum sparse stellatum aeque ac dentes calycis late 
ovati; corolla rotato-campanulata, fere $5 \mathrm{~mm}$ alta, extus sparse stellatotomentosa, lobis orbiculatis tubo brevioribus, stamina limbum superantia; stylus calycis dentes paullo superans. Drupa oblongo-ovoidea, calyce persistente coronata; putamen valde compressum, $8 \mathrm{~mm}$ longum et $5 \mathrm{~mm}$ latum, ventre tri-, dorso bisulcatum.

Hupeh: Kiu Fu, ad ripas flum. Yang-tse-kiang, E. H. Wilson, Veitch Exp. no. 3726 (in herb. Arnold Arb.).

Affine Viburno Bockii Graebner quod foliis subtus cinereo-tomentosis, supra nitentibus minoribus facile distinguitur.

3. Viburnum congestum Rehder, l. c., p. 111.

Frutex circa $1,30 \mathrm{~m}$ altus ramulis usque ad tertium annum tomento steliato denso canescente obtectis. Gemmae nudae. Folia coriacea, persistentia, ovalia $\mathrm{v}$. elliptico-ovata, obtusa $\mathrm{v}$. acuta, basi rotundata v. late cuneata, integra, supra saturate viridia, primo sparse stellato-pubescentia mox glabrescentia, venis leviter impressis, subtus tomento cinereo-albido v. flavo-albido obtecta, $2-4 \mathrm{~cm}$ longa; petioli dense tomentosi, $0,5-$ $1 \mathrm{~cm}$ longi. Corymbi terminales et laterales, densi et parvi, $2-3 \mathrm{~cm}$ diam., pedunculo stellato-tomentoso, $5-10 \mathrm{~mm}$ longo; radii 5 , breves, angulares, stellato-tomentosi; flores subsessiles in radiis primi secundique ordinis, calycis dentes breves et late ovati ovariumque glabra; corolla campanulato-infundibuliformis, $5 \mathrm{~mm}$ longa, glabra, lobis orbiculato-ovatis, tubum circa dimidium aequantibus; stamina lobos aequantes; stylus dentes calycis superans. Fructus desiderantur.

Yunnan: Mengtze, $1600 \mathrm{~m}$ alt., A. Henry no. 9683a (in herb. Kew.); Tapin-tze, Delavay no. 929 (in herb. Kew.).

Arcte affine Viburno utili Hemsl, quol tamen corolla majore fere rotata, inflorescentia majore et laxiore, tomento squamis fulvis intermixtis differt.

4. Viburnum utile Hemsley var. elaeagnifolium Rehder, I. c., p. 89.

A typo differt ramulis gracilioribus, fulvo-tomentosis, foliis tenuioribus, oblongo-lanceolatis, graciliter petiolatis, supra flavo-viridibus, corymbo minore paucifloro, circa $3 \mathrm{~cm}$ diam., fructibus in radiis secundi ordinis.

Hupeh occ., E. H. Wilson, Veitch Exp. no. 31 in parte, quoad ramum fructiferum (in herb. Arnold Arb.).

Sect. Megalotinus Maximowicz.

5. Viburnum crassifolium Rehder. 1. c., p. 112.

Frutex circa $1 \mathrm{~m}$ altus, ramulis angulatis, hornotinis fasciculato-pilosis, annotinis glabris et cinereo- v. rubro-brunneis. Gemmae biperulatae. Folia coriacea, persistentia, elliptica, acuta acumine plerumque torto, basi late cuneata, integra, supra luteo-viridia glabra, subtus pallidiora glandulis minutis sparsis instructa, in axillis venarum fasciculato-barbata, ceterum glabra. utrinque 4-5-costata costis curvatis et anastomosantibus, supra leviter impressis, subtus elevatis, 4-7,5 cm longa et 2,5$3.5 \mathrm{~cm}$ lata; petioli crassi, glabri v. sparse pilosi, $1-1,5 \mathrm{~cm}$ longi. Corymbus terminalis, $4-5 \mathrm{~cm}$ diam., pedunculo angulato, $1-1,5 \mathrm{~cm}$ longo sustentus; radii 7 , angulati. pilis fasciculatis sparsis instructi; 
flores desiderantur. Drupae in radiis secundi ordinis, sessiles, coeruleonigrae, ovoideae, $5 \mathrm{~mm}$ longae, calyce persistente coronatae; putamen leviter compressum, ventre leviter uni-, dorso leviter bisulcatum.

Yunnan, Mengtze in montibus graminosis, $1800-2000 \mathrm{~m}$ alt., A. Henry no. 9797 (in herb. 'Arnold Arb.).

Affine Viburno cylindrico Hamilt. quod corymbo glabro, foliis majoribus nitentibus, statu arboreo facile distinguitur.

6. Viburnum pyramidatum Rehder, 1. c., p. 93, tab. 144.

Arbor $6-7 \mathrm{~m}$ alta ramulis teretibus hornotinis pilis fulvescentibus fasciculatis et stellatis instructis, annotinis glabrescentibus flavo-cinereis, Gemmae biperulatae. Folia chartacea, ovato-oblonga v. oblonga, acuminata, basi cuneata, denticulato-serrata, supra obscure viridia glabra nitentia, subtus pallidiora, laxe fasciculato-pubescentia praesertim ad venas, utrinque 6-7-costata costis arcuatis anastomosantibusque, 8$19 \mathrm{~cm}$ longa et 4-8 cm lata; petioli dense fasciculato-pubescentes, $1,5-$ $2,5 \mathrm{~cm}$ longi. Corymbus terminalis, pyramidalis, $5-6 \mathrm{~cm}$ altus et diam., verticillis 3-4 distantibus apicem versus decrescentibus compositus, verticillo quoque radiis $4-6$ instructo, pedunculo $4-6 \mathrm{~cm}$ Jongo, dense fasciculato-piloso aeque ac radii; flores in radiis tertii ordinis; ovarium cylindricum glabrum; calycis dentes ovati, dimidium ovarium aequantes, sparse ciliati; corolla rotata, $4 \mathrm{~mm}$ diam.. lobis ovatis stamina paullo superantibus; stylus cylindricus calycis dentes superans. Drupa oblonga, circa $1 \mathrm{~cm}$ longa et $\mathbf{4}-\mathbf{5} \mathrm{mm}$ lata, calyce persistente coronata, e sicco obscure rubra; putamen compressum et leviter curvatum, ventre bi-. dorso leviter unisulcatum, testa seminis glandulis rubris resinosis dense obtecta, albumine ruminato.

Yunnan: Mengtze, A. Henry no. 11475.

Affine Viburno lutescenti Blume quod ramulis et foliis subtus glabris satis distincta videtur.

7. Viburnum ternatum Rehder, l. c., p. 37, tab. 117 (1907).

Frutex $1,5-4 \mathrm{~m}$ altus ramulis hornotinis adpresse pubescentibus, vetustioribus griseo-brunneis. Folia ternata v. in ramulis tenuioribus opposita, membranacea, elliptica v. oblongo-obovata, acuta v. subacuminata, basi cuneata, integra, supra lutescenti-viridia glabra, subtus pallidiora glabra pilis longis adpressis in costa media lateralibusque exceptis, utrinque 5-7-costata costis arcuatis anastomosantibusque et trabeculis parallelis conspicuis conjunctis, 8-22 $\mathrm{cm}$ longa et $4-9,5 \mathrm{~cm}$ lata; petioli graciles, $2-5 \mathrm{~cm}$ longi, pubescentes. Corymbus sessilis. laxus, 12-17 cm diam., sparse pubescens, 6-7-radiatus radio medio brevissimo; flores sessiles $v$. brevipedunculati, ultimi in radiis sixti ordinis; ovarium obconicum, vix $1 \mathrm{~mm}$ longum, glabrum; dentes calycis minuti, interdum obsoleti, ciliolati; corolla rotato-campanulata, flavido-alba, $5 \mathrm{~mm}$ diam., lobis semiorbicularibus, $1,5 \mathrm{~cm}$ longis tubum aequantibus; stamina corolla plus quam duplo longiora, $6-7 \mathrm{~mm}$ longa. Drupa rubra, ovoideooblonga; putamen ovoideo-oblongum. 6-7 mm longum ot fere $4 \mathrm{~mm}$ latum, cinereo-album, dorso leviter uni-, ventre obscure bisulcatum, testa seminis punctulata, fusco-rubra. 
China occ., E. H. Wilson, Veitch Exp. no. 3736, 3736a (in herb. Arnold Arb.).

Affine $V$. sambucino Blume quod praecipue, foliis persistentibus, semper oppositis, corymbo pedunculato, densius pubescente differt.

8. Viburnum amplifolium Rehder. 1. c., p. 112.

Frutex 3-4-metralis ramulis junioribus laxe stellato-tomentosis, annotinis glabris fulvescentibus. Gemmae perulis 2 exterioribus stellatotomentosis. Folia membranacea, ovalia v. ovata v. elliptico-ovata, acuminata, basi rotundata $v$. late cuneata, dentata ima basi excepta, supra obscure viridia tuberculis minutis initio pilos furcatos caducos gerentibus asperata, subtus pallidiora glabra pilis sparsis stellatis ad venas venulasque exceptis, utrinque 7-9-costata costis partim anastomosantibus partimque in dentes exeuntibus, $8-14 \mathrm{~cm}$ longa et $6-8,5 \mathrm{~cm}$ lata; petioli canaliculati, fulvescenti-stellato-tomentosi. Corymbi in apice ramulorum lateralium brevium bifoliorum pedunculo stellato-tomentoso, $4-5 \mathrm{~cm}$ longo sustenti; radii 4-6. graciles, cirea $2,5 \mathrm{~cm}$ longi, stellato-tomentosi; flores ignoti. Drupae in radiis secundi et tertii ordinis, ovoideooblongae, $8 \mathrm{~mm}$ longae et $5 \mathrm{~mm}$ latae, calyce styloque persistenti coronatae, stylo cylindrico sepalis ovatis v. ovato-oblongis paullo longiore; putamen ventre profunde sulcatum, dorso leviter bisulcatum.

Yunnan: Mengtze, $2000 \mathrm{~m}_{\mathrm{i}}$ alt., A. Henry no. 13470 (in herb. Arnold Arb. et herb. Kew.).

Proximum V. Colebrookiano Wallich videtur quod ramulis floriferis aphyllis, drupis minoribus, foliis angustioribus, chartaceis, dense serratis, nec dentatis, costis lateralibus semper anastomosantibus facile distinguitur.

9. Viburnum tomentosum var. lanceatum Rehder, l. c., p. 109.

Folia ramulorum floriferorum elliptico-ovata v. elliptico-lanceolata, acuta $v$. acuminata, basi late cuneata $v$. rotundata, $3,5-7 \mathrm{~cm}$ longa ot $1,5--3 \mathrm{~cm}$ lata, supra glabra v. subglabra, subtus sparse stellato-pubescentia, utrinque 6-9-costata; folia turionum lanceolata, acuminata, 5$10 \mathrm{~cm}$ longa, supra pilis furcatis instructa demum glabrescentia, subtus densius stellato-pubescentia, costis paucioribus, petiolis stellato-pubescentibus. Corymbi quam in typo minores floribus sterilibus paucioribus; drupa sex mm longa; ceterum ut in typo.

Japonia: Hondo, prope Nikko, Sept. 8, 1892, C. S. Sargent (in herb. Arnold Arb.). - Cultum in Arboreto Arnoldiano.

Differt a typo omnibus partibus minoribus, foliis angustioribus, magis pubescentibus costis paucioribus et a var. parvifolio (Miqu.) Rehder ( $V$. tomentosum var. cuspidatum Maxim.) praecipue folis sensim acuminatis, nec abrupte cuspidatis, angustioribus.

Viburnum tomentosum Thurib. et $V$. Hanceanum Maxim. in conspectu meo specierum Asiae orientalis ad sectionem Pseudotinum attuleram (1. c.. p. 112), sed nunc mihi persuasum habeo ambas has species melius ad Wegalotinum referendas esse in qua sectione praecipue $V$. Colebrookianum et $V$. amplifolium affinitatem artiorem praebent.

Sect. Tinus Maxim. 
10. Vỉburnum cinnamonifolium Rehder, 1. c.. p. 31, tab. 114 (1907).

Frutex v. arbor glaberrima ad $6 \mathrm{~m}$ alta ramulis fusco-purpureis lenticellis numerosis instructis. Folia coriacea sempervirentia, ellipticooblonga. longe acuminata, basi cuneata, integra v. apicem versus dentibus paucis instituta, basi manifeste trinervia, supra obscure flavo-viridia, subtus pallidiora, $8-13 \mathrm{~cm}$ longa et $3-4,5 \mathrm{~cm}$ lata; petioli satis erassi, $1-2,5 \mathrm{~cm}$ longa. Corymbus terminalis, amplus laxusque, $12-17 \mathrm{~cm}$ diam., pedunculo $2-3 \mathrm{~cm}$ longo sustentus, radiis $6-8$; flores graciliter pedicellati in radiis tertii ordinis; ovarium turbinatum, calycis dentes somiorbiculares $\mathrm{v}$. triangulares, $0,5 \mathrm{~mm}$ longi; corolla viridescenti-alba, rotata, 4-5 mm diam., lobis late ovatis, revolutis, tubum subaequantibus; stamina corolla paullo longiora. Drupa ovoidea, stylo calyceque coronata, atrocyanea nitens, $4 \mathrm{~mm}$ alta et $3 \mathrm{~mm}$ diam., pericarpio vix carnoso; putamen subglobosum, laeve, flavescenti-album, testa seminis fusco-rubra, albumine valde ruminata.

Szech'uan: Mons Omei, E. H. Wilson, Veitch Exp. no. 5022 (in herb. Arnold Arb.).

Arcte affine Viburno Davidii Franchet quod autem foliis crassioribus supra rugosis, corymbo densiore et minore, staminibus brevioribus, calycis dentibus longioribus, drupis majoribus facile distinguitur.

Sect. Odontotinus Rehder, l. c., p. 113.

Frutices indumento fasciculato, gemmis 4-perulatis, foliis plerumque deciduis, dentatis venis rectis in dentes exeuntibus, drupis rubris, putamine compresso leviter sulcato, albumine solido. Pro typo huius sectionis $V$. dilatatum Thunberg inserviatur. (Ser. Dilatata Maximowicz, Mél. Biol., X, 663, 1880).

11. Viburnum theiferum Rehder, 1. c., p. 45, tab. 121 (1907).

Frutex erectus ramulis hornotinis pallide flavo-cinereis, vetustioribus cinereo-brunneis, glabris. Gemmae oblongo-ovoideae, glabrae, circa $6 \mathrm{~mm}$ longae perulis 4 exterioribus. Folia decidua, chartacea v. membranacea, ovato-oblonga, acuminata, basi rotundata, remote denticulata, supra obscure viridia, glabra, subtus pallidiora, glabra pilis longis sericeis ad venas exceptis, utrinque 6-8-costata costis rectis in dentes exeuntibus, $7-12 \mathrm{~cm}$ longa et $3-5,5 \mathrm{~cm}$ lata; petioli glabri v. sparse pubescentes, $1-1,5 \mathrm{~cm}$ longi. Corymbus glaber v. sparse pubescens, $2,5-3,5 \mathrm{~cm}$ diam., pedunculo $1-2,5 \mathrm{~cm}$ longo; radii 3-6-, plerumque 5-flori floribus subsessilibus vel pedicellatis in radiis secundi ordinis; ovarium purpurascens, subcylindrieum, vix $1 \mathrm{~mm}$ longum; dentes calycis ovati, dimidium ovarium aequantes; corolla campanulato-rotata, $5-6 \mathrm{~mm}$ diam., lobis ovatis, $2,5 \mathrm{~mm}$ longis, tubo longioribus; stamina paullo infra limbum inserta, limbum dimidium v. totum aequantia. Drupa rubra, globosoovoidea, calyce persistent coronata; putamen ovoideum, compressum, $8-10 \mathrm{~mm}$ longum et 6-7 mm latum flavo-album, testa rubro-brunnea minute punctulata.

Szechuen: Kui, E. H. Wilson, Veitch Exp. no. 579; Changyang, E. H. Wilson, Veitch Exp. no. 644, S. Wushan, A. Henry no. 5586; Mons Omei, E. Faber (ex Hemsley). 
Affine V. phlebotricho Sieb. ot Zucc. quod foliis circa dimidio minoribus, serratis, brevissime petiolatis, corymbis paucifloris, staminibus brevioribus facile distinguitur. - Infusione foliorum huius fruticis monachi monasteriorum montis Omei pro potu utuntur.

12. Viburnum hirtulum Rehder, I. c., p. 115.

Frutex ramulis junioribus pilis fasciculatis ferrugineis dense obtectis. vetustioribus glabris griseo-brunneis. Folia coriacea, ovata, acuta vel acutiuscula, basi rotundata, remote et obscure denticulata, supra sparse pilis fasciculatis furcatisque instructa, subtus densius praecipue ad venas fasciculato-pilosa, utrinque venis $5-6$ in dentes exeuntibus, supra leviter impressis subtus elevatis, 4-6 cm longa; petioli estipulati, pilis fulvis fasciculatis dense obtecti. Corymbus terminalis, $6-7 \mathrm{~cm}$ diam., aeque ac pedunculi $1-2 \mathrm{~cm}$ longi pilis fasculatis patentibus dense vestitus, radiis $5-6$; flores in radiis tertii $v$. quarti ordinis, basi bracteis lineari bus pubescentibus suffulti; ovaria parva et sepala ovata fasciculatopilosa; corolla rotata, $4-5 \mathrm{~mm}$ diam., extus pubescens lobis orbicularibus ; stamina corollam subaequantia; stylus crassus calycis lobis vix brevior. Fructus desiderantur.

Kwangtung: North River, Nov. 1888, Herb. Hongkong Bot. Gard . no. 116 (in herb. Kew.).

Affine Viburno Mullaha Hamilton et V. Fordiae Hance, prior foliis elliptico-lanceolatis, longe acuminatis, basi cuneatis, supra glabris, corolla parce pilosa, posterior foliis elliptico- v. rhombico-ovatis, basi late cuneatis, supra glabris facile distinguitur.

13. Yiburnum Wilsonii Rehder, 1. c., p. 115.

Frutex ramulis junioribus fasciculato-pilosis, annotinis fusco-purpureis. Gemmae perulis 4 exterioribus fasciculato-pilosis. Folia membranacea ovata, longe acuminata, basi rotundata $v$, late cuneata, fere e basi serrata, supra flavo-viridia pilis furcatis $v$. simplicibus sparsis instructa, subtus pallidiora glabra pilis longis adpressis ad venas exceptis, utrinque venis $6-7$ in dentes exeuntibus, $4-8 \mathrm{~cm}$ longa et $2,5-3 \mathrm{~cm}$ lata; petioli estipulati, $1-1.5 \mathrm{~cm}$ longi, pilis brevibus fasciculatis et longior $\mathrm{j}-$ bus simplicibus instructi. Corymbus terminalis, circa $5 \mathrm{~cm}$ diam., flavescenti-velutinus aeque ac pedunculus circa $2 \mathrm{~cm}$ longus; radii plerumque sex; flores in radiis secundi et tertii ordinis; ovaria $1 \mathrm{~mm}$ longa et sepala ovata pubescentia; corolla rotata extus velutina, $5 \mathrm{~mm}$ diam., lob is orbiculari-ovatis; stamina lobis corollae breviora; stylus crassus et brevis. Fructus desiderantur.

Szechuan: Mons Omei. E. H. Wilson. Veitch Exp. no. 5025 (in herb. Arnold Arb.).

Proximum Viburno Fordiae Hance et V. Mullaha Hamilton quae indumento fasciculato-piloso inflorescentiae, foliis subtus pubescentibus bene distincta.

14. Viburnum ovatifolium Rehder, 1. c., p. 115.

Frutex dichotome ramosus ramulis junioribus glabris, annotinis fusco-purpureis. Gemmae perulis 4 exterioribus. Folia membranacea. 
ovata v. oblongo-ovata, longe acuminata, basi rotundata, serrata, supra obscure flavo-viridia et glabra, subtus pallidiora et glabra pilis simplicibus laxe adpressis ad venas exceptis, utrinque 5-7-costata costis rectis supra leviter impressis in dentes mucronulatos exeuntibus, $5-7 \mathrm{~cm}$ longa et 2,5-4 cm lata; petioli fere glabri, $1-1,5 \mathrm{~cm}$ longi, stipulis subulat is pubescentibus. Corymbus terminalis, $4-6 \mathrm{~cm}$ diam., laxe pilis stellatis minutis longioribus simplicibus intermixtis instructus, pedunculo quam 1 crn breviore; radii $5-7$; flores in radiis tertii ordinis; ovaria laxe stellato-pubescentia, $1 \mathrm{~mm}$ longa; calycis lobi late triangulari-ovati, stellato-pubescentes, $1 \mathrm{~mm}$ longa; corolla rotata, $5 \mathrm{~mm}$ diam., extus sparse fasciculato-pilosa, lobis semiorbicularibus; stamina corolla breviora; stylus brevis calycis dentes paullo superans. Fructus desiderantur.

Yunnan: Mengtze, „north mountains, forests", $3300 \mathrm{~m}$ alt., A. Henry no. 1021a and $1021 \mathrm{~b}$ (in herb. Arnold Arb.).

Affine $V$. eroso Thunb, et $V$. betulifolio Batal: prior facile petiolis brevissimis, foliis acutis $v$, breviter acuminatis, plus minus pubescentibus, staminibus corolla longioribus, posterior petiolis longioribus, folis plerumque late cuneatis, staminibus corollam superantibus, corymbo amplo et laxo distinguitur. Simile etiam est V. luzonicum var. formosanum (Hance) Rehd. quod praecipue petiolis estipulatis brevioribus, foliis breviter acuminatis, plus minus pubescentibus differt.

15. Viburnum dasyanthum Rehder, 1, c., p. 103, tab. 149.

Frutex $2,5 \mathrm{~m}$ altus ramulis junioribus glabris, annotinis castaneis v. fusco-purpureis. Gemmae perulis 4 exterioribus. Folia membranacea, ovata $v$, elliptica $v$, oblonga, longe acuminata, basi rotundata, remote denticulata, supra obscure viridia glabra, subtus pallidiora et fere glabra pilis sparsis ad venas et axillis barbatis exceptis, utrinque 6-7-costata costis rectis in dentes exeuntibus, $6-12 \mathrm{~cm}$ longa et $2,5-3,5 \mathrm{~cm}$ lata; petioli graciles, $1,5-2 \mathrm{~cm}$ longi, glabri rubescentes. Corymbus terminalis, laxus, $8-10 \mathrm{~cm}$ diam., pedunculo glabro, $1-3 \mathrm{~cm}$ longo; radii 7 , interdum 5, glabri, radioli dense villosi; flores in radiis tertii v. quarti ordinis; ovaria ovoidea, $1 \mathrm{~mm}$ longa, dense villosa aeque ac dentes calycis late ovati; corolla campanulato-rotata, extus dense villosa, lobis ovatis tubo longioribus; stamina corollam superantia. Drupa ovoidea rubra, circa $8 \mathrm{~mm}$ longa; putamen late ovoideum compressum, apice acutum, ventre leviter uni-, dorso leviter bisulcatum, testa seminis laeve rubescens.

China: Hupeh, Patung, E. H. Wilson, Veitch Exp. no. 2218 (in herb. Arnold Arb.).

Proximum V. betulifolio Batalin quod corolla corymboque glabris, foliis basi late cuneatis dentatis facile distinguitur.

16. Viburnum hupehense Rehder, l. c., p. 116.

Frutex ramulis junioribus fasciculato-pilosis, annotinis fusco-purpureis glabrescentibus. Gemmae perulis 4 exterioribus. Folia membranacea, late ovata, acuminata, basi truncata vel subcordata, grosse serrata, dentibus brevibus, acuminatis mucronulatisque, supra laxe fasciculato-pilosa, 
obscure flavo-viridia, subtus pallidiora et densius fasciculato-pilosa praesertim ad venas, utrinque costis $7-8$ rectis, $5-7 \mathrm{~cm}$ longa et $3-$ $6 \mathrm{~cm}$ lata; petioli canaliculati, $1,5-2 \mathrm{~cm}$ longi. dense fasciculato-pilosi, stipulis persistentibus lineari-lanceolatis pubescentibus instructi. Corymbus terminalis, $4-5 \mathrm{~cm}$ diam., pedunculo $2 \mathrm{~cm}$ longo dense fasciculatopiloso; radii plerumque 5, dense fasciculato-pilosi; flores desiderantur. Drupae in radiis secundi et tertii ordinis, ovoideae, rubrae; putamen valde compressum, orbiculato-ovatum, $7 \mathrm{~mm}$ longum et $6 \mathrm{~mm}$ latum, ventre tri-, dorso leviter bisulcatum, testa seminis fusco-rubra, minute punctulata.

China: Hupeh, A. Henry no. 6805 (in Herb. Gray).

Affine $V$. dilatato Thunb. et V. betulifolio Batal.; prior praecipue differt foliis petiolis estipulatis foliis plerumque obovatis, posterior foliis glabris rhombicis v. oblongis.

17. Viburnum ichangense Rehder, 1. c., p. 116.

V. erosum var. ichangense Hemsley, Jour. Linn. Soc., XXII, 352, 1888 (in parte). - V. erosum var. setchuenense Graebner, Bot. Jahrb., XXIX. 589,1901 .

Frutex ramulis gracilibus. junioribus stellato-tomentosis pilosisque, annotinis glabrescentibus pallide griseo-brunneis, vetustioribus fuscis. Gemmae parvae perulis 4 pubescentibus exterioribus. Folia membranacea v. chartacea, ovata v. ovato-lanceolata, acuminata, basi truncata v. subcordata, serrata, supra flavo-viridia, pilis fasciculatis v. furcatis tuberculis minutis insidentibus conspersa, subtus stellato-tomentosa, praecipue ad venas, ad costam mediam insuper pilis laxe adpressis instructa, utrinque costis $6-9$ rectis, $3,5-6,5$ longa et $1,2-3 \mathrm{~cm}$ lata; petioli pubescentes, $3-7 \mathrm{~mm}$ longi, stipulis persistentibus subulatis. Corymbi terminales et laterales, $2-4 \mathrm{~cm}$ diam., pubescentes, pedunculis $1-2 \mathrm{~cm}$ longis; radii $4-5$, aeque ac radioli et flores bracteis linearibus pubescentibus suffulti; flores in radiis primi secundique ordinis; ovaria obovoidea, 1,5 $\mathrm{mm}$ longa, stellato-tomentosa aeque ac calycis dentes late ovati; corolla rotata, $5 \mathrm{~mm}$ diam., glabra, lobis orbiculato-ovatis; stamina corollam subaequantia v. ea paullo breviora; stylus brevis, conicus, dentes calycis aequans. Drupa ovoidea, 6-7 mr longa, rubra, plerumque sparse stellato-pubescens, calyce persistenti coronata; putamen $6 \mathrm{~mm}$ longum et $4,5 \mathrm{~mm}$ latum, compressum, dorso leviter bi-, ventre leviter trisulcatum, testa seminis fusca.

China: Hupeh, A. Henry no. 232, 1888, 2289, 5271, 5476, 6594; Szech'uan, A. Henry no. 5276, 7052, A. von Rosthorn no. 2298, 2299.

Proximum $r$, eroso Thunb. quod ovariis glabris, corymbis majoribus multifloris, foliis majoribus ovatis, plerumque acutis differt. 